\title{
Transtorno do Espectro Autista e Síndrome de Savant: Um paradoxo real entre déficit cognitivo e genialidade
}

\author{
Autistic Spectrum Disorder and Savant Syndrome: A real paradox between cognitive deficit and \\ genius \\ Trastorno del espectro autista y síndrome de Savant: Una paradoja real entre déficit cognitivo y \\ genio
}

\section{Resumo}

Introdução: O transtorno do Espectro Autista (TEA) expressa-se na infância, sendo sua característica um atraso no desenvolvimento do indivíduo, interações sociais anômalas, e ainda pode estar presente uma deficiência intelectual. Neste transtorno pode estar incluído a Síndrome de Savant, caracterizada por talento notável em um ou mais domínios, como memória, música, arte, matemática. Objetivo: abordar a relação entre TEA e Síndrome de Savant, uma situação rara na qual uma pessoa com déficit intelectual tem um grande grau de genialidade e sabedoria, tendo muitas habilidades através da sua extraordinária memória. Metodologia: Trata-se de uma pesquisa descritiva do tipo revisão integrativa da literatura, que busca evidências sobre a relação entre TEA e Síndrome de Savant. A pesquisa foi realizada por meio de bases de dados PubMed, MEDLINE, Scielo, CDSR, Google Scholar, BVS e EBSCO, no período de 2000 a 2021. Dessa maneira, totalizaram-se 21 produções científicas para a revisão integrativa da literatura. Resultados: Não há consenso sobre exatamente como as habilidades sábias são usadas em pacientes TEA. Alguns estudos mostram que os "savants" não possuem diferenças na inteligência padrão em comparação com outros indivíduos portadores de TEA. Portanto, pode ser que suas habilidades se desenvolvam simplesmente por meio de muitas horas de prática prolongada. Conclusão: As habilidades "savants" em si pode não ser tão relevantes, haja vista que o comportamento é realizado de forma repetitiva. É importante explorar ainda mais como esses fatores podem influenciar como diferentes habilidades, que podem ser um próximo passo importante na compreensão das habilidades sábias.

Palavras-chave: Transtorno do espectro autista; Síndrome de Savant; Habilidades; Talento. 


\begin{abstract}
Introduction: The Autistic Spectrum Disorder (ASD) is expressed in childhood, its characteristic being a delay in the individual's development, anomalous social interactions, and an intellectual disability may still be present. This disorder may include Savant Syndrome, characterized by remarkable talent in one or more domains, such as memory, music, art, mathematics. Objective: To address the relationship between ASD and Savant Syndrome, a rare situation in which a person with intellectual deficit has a great degree of genius and wisdom, having many abilities through his extraordinary memory. Methodology: This is a descriptive research of the integrative literature review type, which seeks evidence on the relationship between ASD and Savant Syndrome. The search was performed using PubMed, MEDLINE, Scielo, CDSR, Google Scholar, BVS and EBSCO databases, from 2000 to 2021. Thus, 21 scientific productions were totaled for the integrative literature review. Results: There is no consensus on exactly how wise skills are used in ASD patients. Some studies show that savants have no difference in standard intelligence compared to other individuals with ASD. Therefore, it may be that your skills develop simply through many hours of prolonged practice. Conclusion: The skills "savants" themselves may not be as relevant, given that the behavior is performed repetitively. It is important to explore further how these factors can influence how different skills can be an important next step in understanding wise skills.
\end{abstract}

Keywords: Autistic spectrum disorder; Savant Syndrome; Skills; Talent.

\title{
Resumen
}

Introducción: El Trastorno del Espectro Autista (TEA) se expresa en la niñez, siendo su característica un retraso en el desarrollo del individuo, interacciones sociales anómalas y una discapacidad intelectual que aún puede estar presente. Este trastorno puede incluir el síndrome de Savant, caracterizado por un talento notable en uno o más dominios, como la memoria, la música, el arte, las matemáticas. Objetivo: Abordar la relación entre el TEA y el Síndrome de Savant, una situación poco común en la que una persona con déficit intelectual tiene un gran grado de genio y sabiduría, teniendo muchas habilidades a través de su extraordinaria memoria. Metodología: Se trata de una investigación descriptiva del tipo revisión integradora de la literatura, que busca evidencia sobre la relación entre TEA y Síndrome de Savant. La búsqueda se realizó mediante las bases de datos PubMed, MEDLINE, Scielo, CDSR, Google Scholar, BVS y EBSCO, de 2000 a 2021. Así, se totalizaron 21 producciones científicas para la revisión integradora de la literatura. Resultados: No hay consenso sobre exactamente cómo se utilizan las habilidades inteligentes en los pacientes con TEA. Algunos estudios muestran que los sabios no tienen diferencias en la inteligencia estándar en comparación con otros individuos con TEA. Por lo tanto, es posible que sus habilidades se desarrollen simplemente a través de muchas horas de práctica prolongada. Conclusión: Las habilidades "sabias" en sí mismas pueden no ser tan relevantes, dado que el comportamiento se realiza de manera repetitiva. Es importante explorar más a fondo cómo estos factores pueden influir en cómo las diferentes habilidades pueden ser un próximo paso importante en la comprensión de las habilidades inteligentes.

Palabras clave: Trastorno del espectro autista; Síndrome de Savant; Habilidades; Talento.

\section{Introdução}

O transtorno do espectro autista (TEA) representa um conjunto heterogéneo de condições do neurodesenvolvimento, caracterizadas por dificuldades na comunicação social, bem como comportamentos e interesses repetitivos e restritivos de instalação precoce. O TEA é uma patologia compreendida de forma incompleta, não existindo nenhuma etiologia específica cuja modificação culmine invariavelmente no seu surgimento. No entanto, as várias alterações possíveis tornam-na numa doença que resulta de insultos multifatoriais dinâmicos em diferentes fases do desenvolvimento, que precedem e produzem heterogeneidade fenotípica. As mutações genéticas, alterações epigenéticas e fatores de risco imunológicos são as etiologias que condicionam as alterações fisiopatológicas desta patologia (Eissa et al., 2018).

As alterações fisiopatológicas do TEA podem ser agrupadas ainda em morfológicas, funcionais e bioquímicas, estando estas interligadas. Modificações bioquímicas nas vias de sinalização mais relevantes e nos diferentes neurotransmissores traduzem-se nas respetivas alterações funcionais (tanto excesso de ativação como inibição), que, por sua vez, impactam nas alterações morfológicas ao longo do neurodesenvolvimento e no próprio cérebro já formado dos indivíduos afetados, assim como na conectividade funcional entre diversas regiões (Subramayan et al., 2019).

Uma pequena parcela de pessoas com transtorno do espectro autista (TEA) desenvolve uma espécie de genialismo, ou seja, pessoas muito inteligentes que possuem habilidades extraordinárias. Acredita-se que a disfunção de determinadas regiões cerebrais (como o TEA) provoque uma resposta paradoxal com a super ativação e potencialização de outras áreas do cérebro. 
Esse processo é chamado de "facilitação funcional paradoxal" e foi descrito por Kapur, em 1996. Desta maneira, após haver um dano cerebral em determinada região, geralmente no hemisfério esquerdo, há ativação de outra região cerebral, com reconfiguração da circuitaria neural e o aparecimento de capacidades até então adormecida, e isso ocorre por um processo de desinibição de habilidades previamente armazenadas na nova região recrutada (Efstratopolou, 2017).

No entanto, o surgimento de habilidades "savant" em adultos autistas não é totalmente compreendido, e há uma falta de evidências empíricas para apoiar as teorias atuais. Desse modo, a motivação para a pesquisa atual é investigar a natureza e grau de frequência das habilidades "savant" em indivíduos com TEA, bem como a relação entre as habilidades cognitivas excepcionais e presença de comportamentos obsessivos e repetitivos.

\section{Metodologia}

Trata-se de uma pesquisa descritiva do tipo revisão integrativa da literatura, que buscou responder quais as evidências sobre a relação entre TEA e Síndrome de Savant. A pesquisa foi realizada através do acesso online nas bases de dados National Library of Medicine (PubMed MEDLINE), Scientific Electronic Library Online (Scielo), Cochrane Database of Systematic Reviews (CDSR), Google Scholar, Biblioteca Virtual em Saúde (BVS) e EBSCO Information Services, no mês de julho de 2021.

Para a busca das obras foram utilizadas as palavras-chaves presentes nos descritores em Ciências da Saúde (DeCS): em inglês: "autism spectrum disorder", "savant syndrome", "genius", "skills", em português: "transtorno do espectro autista", "síndrome de savant", "genialidade", "habilidades", e em espanhol: "trastorno del espectro autista", "síndrome de savant", "genio", "habilidades".

Como critérios de inclusão, foram considerados artigos originais, que abordassem o tema pesquisado e permitissem acesso integral ao conteúdo do estudo, publicados no período de 2000 a 2021, em inglês, português e espanhol. O critério de exclusão foi imposto naqueles trabalhos que não estavam em inglês, português ou espanhol, que não tinham passado por processo de Peer-View e que não relacionassem o TEA e a Síndrome de Savant.

A estratégia de seleção dos artigos seguiu as seguintes etapas: busca nas bases de dados selecionadas; leitura dos títulos de todos os artigos encontrados e exclusão daqueles que não abordavam o assunto; leitura crítica dos resumos dos artigos e leitura na íntegra dos artigos selecionados nas etapas anteriores. Após leitura criteriosa das publicações, 6 artigos não foram utilizados devido aos critérios de exclusão. Assim, totalizaram-se 21 artigos científicos para a revisão integrativa da literatura, com os descritores apresentados acima. Após esta seleção, filtraram-se por artigos dos últimos vinte e um anos e por artigos em línguas portuguesa, inglesa e espanhola.

\section{Resultados e Discussão}

Após a seleção dos artigos por meio dos critérios de inclusão e de exclusão, elaborou-se uma tabela (Tabela 1) contendo as principais informações sobre a associação entre TEA e Síndrome de Savant. A análise permitiu a percepção de que os estudos sugerem essas duas patologias podem estar intimamente associadas. 
Tabela 1: Estudos utilizados na Revisão Integrativa e achados principais.

\begin{tabular}{|c|c|c|}
\hline AUTOR & ANO & PRINCIPAIS ACHADOS \\
\hline $\begin{array}{l}\text { Rodrigues, V., et } \\
\text { al. }\end{array}$ & 2020 & $\begin{array}{l}\text { No padrão de competências da Síndrome de Savant, as mais comuns são competências divididas, que } \\
\text { incluem uma preocupação obsessiva com memorização de músicas, placas de carros, mapas, fatos } \\
\text { históricos ou itens mais específicos, como o som de um motor ou de um aspirador. }\end{array}$ \\
\hline $\begin{array}{l}\text { Ataíde, B. D. G., } \\
\text { et al. }\end{array}$ & 2017 & $\begin{array}{l}\text { A habilidade do paciente com Síndrome de Savant e TEA pode estar relacionada ao fato desses indivíduos } \\
\text { passarem muito tempo analisando calendários, o que denota uma grande capacidade de memória ou de } \\
\text { uma capacidade extraordinária e inconsciente de ligar datas a partir do conhecimento da regularidade do } \\
\text { calendário. }\end{array}$ \\
\hline Martino, G. & 2017 & $\begin{array}{l}\text { Gerenciar o calendário é uma habilidade frequentemente descrita dentro da Síndrome de Savant, que é } \\
\text { caracterizada por memória extraordinária, cálculo com grande velocidade e capacidade de correlacionar } \\
\text { dias, datas e anos. }\end{array}$ \\
\hline Bosa, C., et al. & 2000 & $\begin{array}{l}\text { Diferenças no sistema de processamento da informação em crianças com autismo é também a base da } \\
\text { Síndrome de Savant, haja vista que a falta da tendência natural em juntar partes de informações para } \\
\text { formar um todo provido de significado (coerência central) é uma das características mais marcantes no } \\
\text { autismo. }\end{array}$ \\
\hline Klin, A. & 2006 & $\begin{array}{l}\text { Do ponto de vista neuropsicológico, no indivíduo com TEA existe, em geral, um padrão relativamente } \\
\text { elevado em habilidades auditivas, verbais e aprendizado repetitivo, o que pode atestar as teorias de } \\
\text { correlação com a Síndrome de Savant. }\end{array}$ \\
\hline $\begin{array}{l}\text { Hermelin, B., et } \\
\text { al. }\end{array}$ & 2001 & $\begin{array}{l}\text { Em indivíduos com TEA, a preocupação com os detalhes pode dar-lhes maior senso de organização e } \\
\text { coerência, podendo até melhorar seu desempenho em certas tarefas que penalizam a estratégia configural, } \\
\text { e parecem ser atraídos por partes fragmentadas, em vez "do todo", e às rotinas, em vez da novidade, como } \\
\text { uma forma de evitar ficar sobrecarregado pela complexidade de imagens, o que pode explicar a Síndrome } \\
\text { de Savant. }\end{array}$ \\
\hline $\begin{array}{l}\text { Yunta, J. A. M., } \\
\text { et al. }\end{array}$ & 2003 & $\begin{array}{l}\text { Pacientes com Síndrome de Savant associado ao TEA caracterizam-se por terem um repertório limitado } \\
\text { de habilidades, que geralmente correspondem ao hemisfério esquerdo. No entanto, geralmente são } \\
\text { extremamente hábeis nas atividades que envolvem o hemisfério direito, situação característica de } \\
\text { pacientes com demência frontotemporal e senis, embora não seja o caso da memória. }\end{array}$ \\
\hline Treffert, D. A. & 2014 & $\begin{array}{l}\text { Ainda não se sabe ao certo como as pessoas com um distúrbio neurológico diagnosticado desenvolvem } \\
\text { uma área ou ambiente marcante após o evento traumático (como o TEA), sendo uma das explicações } \\
\text { apresentadas aponta para a teoria da facilitação funcional paradoxal. De acordo com essa teoria, a } \\
\text { Síndrome de Savant, tanto adquirida quanto congênita, provém de dano ou disfunção cerebral em uma } \\
\text { área, geralmente no hemisfério esquerdo, que causa um fortalecimento do tecido cerebral intacto (o } \\
\text { hemisfério direito). Portanto, o cérebro usa esse tecido como um único motor de operação, e é aí que essas } \\
\text { novas habilidades são criadas. }\end{array}$ \\
\hline Dohn, A., et al. & 2012 & Pessoas com Síndrome de Savant tendem a exibir criatividade e imaginação musical acima da média. \\
\hline $\begin{array}{l}\text { Hughes, J. E. A., } \\
\text { et al. }\end{array}$ & 2018 & $\begin{array}{l}\text { Sensibilidade sensorial aumentada, comportamentos obsessivos, sistematização, habilidades técnicas e } \\
\text { espaciais foram todos os aspectos-chave na definição do perfil do indivíduo com Síndrome de Savant } \\
\text { distinto do autismo sozinho, juntamente com uma abordagem diferente para aprendizagem de tarefas. }\end{array}$ \\
\hline Mottron, L., et al. & 2006 & $\begin{array}{l}\text { As habilidades do paciente com Síndrome de Savant podem existir em uma variedade de áreas, mas a } \\
\text { maioria desses indivíduos mostram habilidades em arte (por exemplo, desenhos hiper-detalhados), música } \\
\text { (proficiência em tocar instrumento musical), matemática (rápida aritmética mental), gestão de calendário } \\
\text { (capacidade de fornecer o dia da semana para qualquer data), e evocação da memória de fatos, eventos, } \\
\text { números etc. }\end{array}$ \\
\hline Bölte, S., et al. & 2004 & $\begin{array}{l}\text { O estudo evidenciou que indivíduos com Síndrome de Savant não apresentam diferenças no padrão } \\
\text { inteligência em comparação com outros indivíduos autistas, e suas habilidades especiais podem, portanto, } \\
\text { serem advindas através de muitas horas de prática devido obsessão prolongada por determinada tarefa. }\end{array}$ \\
\hline Fabricius, $\mathrm{T}$. & 2010 & $\begin{array}{l}\text { Savantismo é um pequeno subconjunto do campo do autismo, caracterizado por indivíduos que exibem } \\
\text { uma habilidade impressionante em uma área específica. "Savants" que têm talentos nas artes visuais } \\
\text { podem recriar desenhos, pinturas e esculturas precisas que capturam detalhes minuciosos com um nível } \\
\text { surpreendente de precisão. }\end{array}$ \\
\hline Miller, B., et al. & 2006 & $\begin{array}{l}\text { O estudo notou que a Síndrome de Savant pode ser adquirida após o diagnóstico de autismo, bem como } \\
\text { semelhanças com certos tipos de doenças neurodegenerativas, como demência frontotemporal, achado } \\
\text { ilustrado pela indução temporária de habilidades perceptivas aprimoradas em indivíduos normais através } \\
\text { da aplicação de estímulos transmagnéticos das áreas frontotemporais do cérebro. }\end{array}$ \\
\hline Snyder, A., et al. & 2009 & $\begin{array}{l}\text { Indivíduos com TEA e Síndrome de Savant têm acesso privilegiado a informações de nível inferior e } \\
\text { menos processadas, antes que sejam empacotadas em conceitos holísticos e rótulos significativos. Devido } \\
\text { a uma falha na inibição de cima para baixo, eles podem acessar informações que existem em todos os } \\
\text { nossos cérebros, mas que normalmente estão além da percepção consciente. }\end{array}$ \\
\hline
\end{tabular}




\begin{tabular}{|c|c|c|}
\hline Song, J., et al. & 2019 & $\begin{array}{l}\text { Em comparação com neurônios do grupo controle, os autistas com Síndrome de Savant exibiram níveis } \\
\text { elevados de expressão dos genes ASD, que estão relacionados à dificuldade de aprendizagem. }\end{array}$ \\
\hline Howlin, P., et al. & 2004 & $\begin{array}{l}\text { As habilidades mais comumente relatadas dos indivíduos com TEA e Síndrome de Savant são habilidades } \\
\text { matemáticas (cálculos de calendário, aritmética veloz e cálculos de números primos), música } \\
\text { (especialmente a capacidade de repetir sequências complexas após apenas uma exposição), arte (cenas } \\
\text { complexas com perspectiva precisa e memória para datas, lugares, rotas ou fatos. São relatadas com } \\
\text { menos frequência as habilidades "pseudo-verbais" (hiperlexia ou facilidade com línguas estrangeiras) e } \\
\text { aptidão mecânica. }\end{array}$ \\
\hline $\begin{array}{l}\text { Iavarone, A., et } \\
\text { al. }\end{array}$ & 2007 & $\begin{array}{l}\text { No acompanhamento por Kanner de pacientes com autismo, em 1971, de seus } 11 \text { casos originais, seis } \\
\text { indivíduos foram descritos como tendo uma excelente habilidade (sugestivo de Síndrome de Savant), } \\
\text { principalmente nas áreas de música e memória mecânica. Dois dos quatro casos descritos em detalhes por } \\
\text { Asperger, tiveram excelente habilidades de cálculo; outro tinha ortografia excepcional. }\end{array}$ \\
\hline Treffert, D. A & 2009 & $\begin{array}{l}\text { Ainda não se sabe ao certo como as pessoas com um distúrbio neurológico diagnosticado desenvolvem } \\
\text { uma área específica após o evento traumático. Uma das explicações apresentadas aponta para a teoria da } \\
\text { facilitação funcional paradoxal. Especificamente, foi demonstrado que o feto masculino, ao contrário do } \\
\text { feminino, apresenta níveis muito elevados de testosterona. Esses hormônios em grande quantidade podem } \\
\text { retardar o crescimento e deteriorar a função neuronal do hemisfério esquerdo do cérebro, o que levaria a } \\
\text { um desenvolvimento maior do que o normal do hemisfério direito e seu domínio sobre o esquerdo, que } \\
\text { tem sido visto que eles ocorrem no caso de crianças com TEA e Síndrome de Savant. }\end{array}$ \\
\hline Oblak, A., et al. & 2009 & $\begin{array}{l}\text { Os "savants" autistas retêm toda a representação do sinal sensorial em níveis mais elevados de } \\
\text { cognição. Pessoas cognitivamente típicas absorvem todo o sinal neural, mas os espalham para um } \\
\text { subconjunto menor de neurônios que capturam uma aproximação do sinal conforme indicado pelo sinal, o } \\
\text { que compensa a eficiência por meio de compressão versus retenção de detalhes. }\end{array}$ \\
\hline Ma, D. Q., et al. & 2005 & $\begin{array}{l}\text { Os resultados não apoiam habilidades "savants" como um subconjunto fenotípico informativo para } \\
\text { ligação nesses dados. O uso de habilidades "savant" como uma covariável pode precisar de mais } \\
\text { exploração em outros grupos. A possibilidade de que esta covariável pode ser subsumido em classes de } \\
\text { comportamento maiores (ou seja, insistência em uniformidade) também deve ser considerada. }\end{array}$ \\
\hline
\end{tabular}

Fonte: Autores (2021).

Retratos do transtorno do espectro do autismo em filmes, televisão e a literatura muitas vezes mostra habilidades especiais ou "savants": uma criança que pode quebrar códigos avançados, um adulto com surpreendente memória, ou um músico que pode tocar qualquer música de ouvido após uma única audiência (Oblak et al., 2009). O autismo descreve um conjunto de sintomas tomos envolvendo dificuldades na comunicação social, comportamentos geralmente repetitivos e rotineiros, excepcionalmente estreitos interesses e sensibilidade atípica aos estímulos sensoriais, bem como de condições associativas em áreas como processamento perceptivo e atenção aos detalhes, bem como áreas de aptidão e talentos específicos (Mottron et al., 2006). Dessa forma, na Síndrome de Savant, talentos e habilidades observadas em tais indivíduos excedem em muito a nível geral de funcionamento intelectual ou de desenvolvimento.

Um dos mais fascinantes fenômenos cognitivos no autismo é a presença das denominadas "ilhotas de habilidades especiais", habilidades preservadas ou altamente desenvolvidas em certas áreas que contrastam com os déficits gerais de funcionamento da criança (Hermelin et al., 2001). Não é incomum, por exemplo, que as crianças com autismo tenham grande facilidade de decifrar letras e números, às vezes precocemente (hiperlexia), mesmo que a compreensão do que leem esteja muito prejudicada. Esse fascinante fenômeno relaciona-se a um âmbito reduzido de capacidades, como memorização de listas ou de informações triviais, cálculos de calendários, habilidades visuoespaciais, tais como desenho ou habilidades musicais envolvendo tonalidade musical perfeita ou tocar uma peça musical após tê-la ouvido somente uma vez. É interessante que indivíduos autistas representam uma maioria desproporcional entre todas as pessoas "savants” (Klin, 2006).

As habilidades "savants" mais reconhecidas são as relacionadas à música, artes plásticas, cálculo e matemática, sendo, aqueles com habilidades musicais, "músicos de ouvido", capazes de cantar canções perfeitamente e interpretar as letras com facilidade incrível, bem como podem dominar vários instrumentos e criar suas próprias composições em nunca terem estudado música. O "savant" artista se expressa muito bem com o desenho, pintura e escultura, e são capazes de capturar e trabalhar com grande detalhe (Ataíde, et al., 2017). Pessoas com Síndrome de Savant tendem a exibir criatividade e imaginação musical 
acima da média. Além disso, muitos pesquisadores concordam que existe uma conexão entre o tom absoluto e o savantismo e que, por sua vez, as pessoas que possuem essa capacidade tendem a estar mais próximas do autismo. O "som absoluto" é uma habilidade possuída por alguns desses sujeitos, pela qual eles são capazes de reproduzir uma nota específica sem o auxílio de uma referência musical, seja por instrumentação ou por canto. (Dohn, et al., 2012). No entanto, uma característica muito comum nesses indivíduos, principalmente quando incidentes também com TEA, é a retenção exagerada de lembranças e memória extraordinária.

Segundo Howlin et al. (2004), em uma pesquisa envolvendo 5400 pais de crianças com autismo, descobriram que 531 $(9,8 \%)$ foram relatados como tendo habilidades "savants". Daqueles com habilidades relatadas, as mais comuns foram música (53\%), memória (40\%), habilidades matemáticas e de cálculo (25\%) e arte (19\%), e 53\% tinham múltiplas habilidades especiais. Além disso, de acordo com Iavarone et al. (2007), em estudo com 254 indivíduos com autismo, foram identificados $33(13 \%)$ com pelo menos uma habilidade especial avaliada pelo questionário de habilidades da Síndrome de Savant. Estudo de Yunta et al. (2003) confirma que esses pacientes apresentam um déficit significativo nas habilidades do hemisfério esquerdo, de aspecto lógico e simbólico, bem como de habilidades linguísticas e especiais, no entanto, apresentam aumento em habilidades características do hemisfério direito, como a artística não simbólica, habilidades visuais e motoras, habilidades artísticas, plásticas e musicais.

A habilidade de correlacionar datas aos dias da semana, sejam antigas ou futuras, é ainda um mistério, e alguns teóricos alegam a possibilidade de, devido sua extraordinária memória, se lembrarem de calendários previamente observados, porém, essa hipótese não corresponderia à possibilidade de fornecimento de datas em tempo futuro, haja vista a raridade da existência de calendários deste tipo (Martino, 2017). Além disso, casos excepcionais de Síndrome de Savant prodigioso ocorrem quando o nível de habilidade de um indivíduo autista vai além visto até mesmo na população em geral. Um conhecido exemplo de um sábio prodigioso é o artista Stephen Wiltshire, que é capaz de desenhar cidades hiperdetalhadas escapos de memória e quem também tem autismo (Hughes et al., 2018).

Não há consenso sobre exatamente como as habilidades "savants" são desenvolvidas em indivíduos autistas. Estudo de Bölte et al. (2004) mostrou que os sábios não apresentam diferenças no padrão inteligência em comparação com outros indivíduos autistas. Isto pode, portanto, ser que suas habilidades se desenvolvam simplesmente através de muitas horas de prática prolongada, o que seria semelhante às habilidades de atletas de memória neurotípicos que podem, por exemplo, corroborar na memorização de milhares de dígitos de pi usando técnicas mnemônicas, com os melhores desempenhos contando com muitas horas de prática, como em outras esportes. Além disso, esses indivíduos também parecem exigir prática, no entanto, o porquê de quererem praticar ainda não se sabe ao certo.

Logo, pode existir duas hipóteses através dessa associação entre o savantismo e TEA. A primeira insinua que há uma possibilidade de uma preocupação obsessiva com uma questão específica, como o caso de calendário, música, ou algo de interesse para o indivíduo que o faça prender a atenção, podendo, assim, superar as habilidades dessa área, mas não em outras tarefas, sendo essa justificativa baseada na ideia de que muitos autistas realizam comportamentos obsessivos e repetitivos, o que explicaria a prevalência alta de Síndrome de Savant associada ao TEA, haja vista que isso não é uma característica singular do TEA, já que não são todos esses indivíduos capazes de desenvolver habilidades especiais (Rodrigues et al. 2020). Já a segunda teoria baseia-se no funcionamento psicológico dos indivíduos com autismo, que mostram um processamento de informação caracterizado por uma fraca capacidade de coerência central e atenção focal, o que pode corroborar o desenvolvimento de algumas habilidades "savants", e, dessa forma, as duas hipóteses explicariam a existência de talentos especiais em indivíduos com baixo nível cognitivo (Bosa et al. 2000).

Além disso, de acordo com os estudos mais recentes, acredita-se que a Síndrome de Savant pode ser adquirida e congênita. No caso do savantismo adquirido, sabe-se que é criado após ter sofrido uma lesão cerebral, decorrente de um 
acidente vascular cerebral, um traumatismo craniano ou outra doença ou lesão do Sistema Nervoso Central. Ao apresentar uma lesão desse tipo, o cérebro cria um método de compensação de habilidades, trocando as habilidades cognitivas que já possuíam pelas novas habilidades sábias, neste caso. No entanto, houve casos em que essas habilidades se manifestaram de forma congênita. É o caso do TEA, embora deva-se observar que nem todas as pessoas com esse transtorno são altamente competentes em uma habilidade específica (Treffert, 2014).

Assim, pode ser que pessoas com distúrbio neurológico diagnosticado desenvolvam uma área ou ambiente marcante após o evento traumático, evento que ainda não tem explicação certeira. Uma das hipóteses aponta para a teoria da facilitação funcional paradoxal. De acordo com essa teoria, a Síndrome de Savant, tanto adquirida quanto congênita, provém de dano ou disfunção cerebral em uma área, geralmente no hemisfério esquerdo, que causa um fortalecimento do tecido cerebral intacto (o hemisfério direito). Portanto, o cérebro usa esse tecido como um único motor de operação, e é aí que essas novas habilidades são criadas (Miller et al., 2006). Essa teoria explicaria a incidência de acordo com o sexo da mesma forma. Especificamente, foi demonstrado que o feto masculino, ao contrário do feminino, apresenta níveis muito elevados de testosterona. Esses hormônios em grande quantidade podem retardar o crescimento e deteriorar a função neuronal do hemisfério esquerdo do cérebro, o que levaria a um desenvolvimento maior do que o normal do hemisfério direito e seu domínio sobre o esquerdo que tem sido visto que eles ocorrem no caso de crianças com ASD (Song et al., 2019). Portanto, os estudos realizados mostram diferenças no número de homens e mulheres com TEA e especificamente Síndrome de Savant. Assim, a proporção em TEA seria de 4: 1 de homens em relação às mulheres, e as diferenças aumentariam no caso do savantismo, apresentando uma proporção de 6: 1 (Treffert, 2009).

Segundo hipótese de Snyder et al. (2009), os "savants" têm acesso privilegiado a informações de nível inferior e menos processadas, antes que sejam empacotadas em conceitos holísticos e rótulos significativos. Devido a uma falha na inibição de cima para baixo, eles podem acessar informações que existem em todos os nossos cérebros, mas que normalmente estão além da percepção consciente. Isso sugere por que as habilidades de savant podem surgir espontaneamente em pessoas normais, e por que tais habilidades podem ser artificialmente induzidas por estimulação magnética transcraniana repetitiva de baixa frequência. Também sugere por que autistas savants são atipicamente literais, com tendência a se concentrar mais nas partes do que no todo, e por que isso oferece vantagens para classes específicas de resolução de problemas, como aquelas que precisam quebrar a mentalidade cognitiva.

Já para Fabricius et al (2010), esses indivíduos têm olhos ouvidos melhores para certos detalhes, ou seu cérebro faz algo diferente com o sensor de informações históricas, haja vista que, excluindo qualquer estudo que encontre uma densidade aumentada de fotorreceptores retinais em crianças com autismo, o cérebro é a fonte mais provável da habilidade "savants", sendo a questão duvidosa voltada em como o cérebro do savant teria acesso aos detalhes que parecem iludir indivíduos cognitivamente típicos. Outro problema em potencial seria se as habilidades "savants" representariam um fator independente de rígidos e obsessivos padrões de comportamento encontrados em indivíduos com autismo. Tipicamente, muitas habilidades especiais são parte de um estreito comportamento repetitivo em indivíduos com autismo. Assim, a habilidade "savant" em si pode não ser tão relevante, haja vista que o comportamento é realizado de forma repetitiva ou compulsiva e resiste à mudança (Ma et al., 2005).

\section{Conclusão}

Ao que parece, os danos mentais que os "savants" têm os deixam sem acesso a grande parte da memória consciente, portanto, os cérebros desses indivíduos simplesmente transferem as funções para a memória implícita, o que corrobora habilidades provenientes dessa síndrome, como a facilidade em memorização, sobretudo de itens como catálogo telefônicos e dicionários, rapidez em realizar cálculos sem auxílio de qualquer material de suporte, bem como talentos musicais e artísticos. 
Essas habilidades costumam aparecer na infância, mas também podem surgir em indivíduos neurotípicos após lesão cerebral ou alguma doença que surge mais tarde na infância ou vida adulta.

O diagnóstico da Síndrome de Savant é clínico, e por isso, o médico deve avaliar o histórico do paciente e realizar uma avaliação neuropsicológica, bem como, após avaliará as habilidades, se achar necessário, poderá solicitar exames de imagem para identificar o risco de tumores ou acidentes vasculares cerebrais. Não há cura para a Síndrome de Savant, pois ela não é uma doença, mas há terapias que podem ajudar a aumentar a qualidade de vida, além de minimizar as dificuldades e aumentar outras habilidades, que costumam ser individualizadas, pois consideram os pontos fortes e dificuldades das pessoas com a síndrome.

Portanto, nota-se que existe uma forte relação de prevalência entre TEA e Síndrome de Savant, porém, não é uma simultaneidade obrigatória, por isso, no TEA, a percepção deve ser vista como uma parte integrante dos mecanismos de habilidades "savants", na medida em que essas mesmas habilidades inesperadamente fortes são manifestações intrínsecas de comportamentos autistas, como a aprendizagem e a inteligência. No entanto, ainda não é claro a medida em que os mecanismos perceptuais básicos estão relacionados a elevadas habilidades autistas com habilidades sábias, já que a condição é rara. Dessa forma, é de suma importância que estudos posteriores abrangem este tema, para melhor qualidade empírica do manejo dessa condição associativa.

\section{Referências}

Ataíde, B. D. G., Lima, B. M. Y., Oliveira, I. A., Marques, F. F \& Borges, T. (2017). Síndrome de Savant: um relato de caso da habilidade intelectual fascinante associada a um déficit de inteligência. Revista Educação em Saúde. 5 (1).

Bölte, S. \& Poustka, F. (2004). Escala de observação diagnóstica para transtornos autistas: resultados iniciais de confiabilidade e validade. Z Kinder Jugendpsychiatr Psychother. 32 (1), 45-50. https://doi.org/10.1024 / 1422-4917.32.1.45.

Bosa, C. \& Calias, M. (2000) Autismo: breve revisão de diferentes abordagens. Psicologia: Reflexão e Crítica, 13 (1), 167-177. https://doi.org/10.1590/S010279722000000100017.

Dohn, A., Garza-Villarreal, E. A., Heaton, P., \& Vuust, P. (2012). Do musicians with perfect pitch have more autism traits than musicians without perfect pitch? an empirical study. PLOS ONE, 7 (5), 1-8. https://doi.org/10.1371/journal.pone.0037961

Efstratopoulou, M. (2017) The Enigma of Autism: Genius, Disorder or just different? Nova Sciences Publishers.

Eissa, N., Al-Houqani, M., Sadeq, A., Ojha, S. K., Sasse, A., \& Sadek, B. (2018). Current enlightenment about etiology and pharmacological treatment of autismo spectrum disorder. Frontiers in Neuroscience, 12 (304). https://doi.org/10.3389/fnins.2018.00304

Fabricius, T. (2010). The Savant Hypothesis: Is autism a signal-processing problem? Medical Hypotheses. 75 (1), 257-265. https://doi.org/10.1016/j.mehy.2010.02.034.

Hermelin, B. (2001). Bright splinters of the mind: a personal story of research with autistic savants. Jessica Kingsley Publishers.

Howlin P., Goode S., Hutton J. \& Rutter M. (2004). Adult outcome for children with autism. J Child Psychol Psychiatry. 45 (2), 212-229. https://doi.org/ $10.1111 / \mathrm{j} .1469-7610.2004 .00215$.

Hughues, J. E. A., Ward, J., Gruffydd, E., Cohen, S. B., Smith, P., Allison, C. \& Simner, J. (2018). Savant syndrome has a distinct psychological profile in autism. Molecular autism. 9 (53). https://doi.org/10.1186/s13229-018-0237-1

Iavarone, A., Patruno, M., Galeone, F., Chieffi, S. \& Carlomagno, S. (2007). Breve relatório: padrão de erro em uma calculadora de calendário autista savant. J. Autism Dev. Desordem. 37 (1), 775-779. https://doi.org/10.1007 / s10803-006-0190-2

Klin, A. (2006). Autismo e síndrome de Asperger: uma visão geral. Rev Bras Psiquiatr, 28 (1), 3-11.

Martino, G. (2017). Autismo y fundamentalismo cognitivo: el Síndrome Savant. Revista Pediátrica Elizalde, 8 (1), 13 -19.

Miller, B. \& Hou, C. (2004). Retratos de autistas. Arch Neurol. 61 (1), 842-844.

Mottron, L., Dawson, M., Soulières, I., Hubert, B. \& Burack, J. (2006). Enhanced Perceptual Functioning in Autism: An Update, and Eight Principles of Autistic Perception. Journal of Autism and Developmental Disorders. 36 (1). 27-43. https://doi.org/10.1007/s10803-005-0040-7.

Mu, D. Q., Jaworski, J., Menold, M. M., Donnelly, S., Abramson, R. K., Whright, H> H., Delong, G. R., Gilbert, J. R., Vance, M. A, P. \& Cuccaro M. L. (2005). Ordered-Subset Analysis of Savant Skills in Autism for 15q11-q13. American Journal of Medical Genetics Part B (Neuropsychiatric Genetics). 135 (1), 38-41. 
Research, Society and Development, v. 10, n. 9, e26310918107, 2021

(CC BY 4.0) | ISSN 2525-3409 | DOI: http://dx.doi.org/10.33448/rsd-v10i9.18107

Oblak, A., Gibbs T. T. \& Blatt G. J (2009). Decreased GABA A receptors and benzodiazepine locais de ligação no córtex cingulado anterior no autismo. Autism Res. 2 (4), 205-219. https://doi.org/10.1002/aur.88

Rodrigues, V., Nascimento, S. \& Maia, L. Transtorno do espectro autista e Síndrome de Savant. Psicologia, Saúde e Doenças. 21 (2), $387-394$. http://dx.doi.org/10.15309/20psd210213

Snyder, A. (2009). Explaining and inducing savant skills: privileged access to lower level, less-processed information. Philos Trans R Soc Lond B Biol Sci. 27 (364), 1399-1405. https://doi.org/10.1098/rstb.2008.0290.

Song, J., Yang, X., Zhou, Y., Zhang, X., Liu, Z., Niu, W., Zhan, N., Fan, X., Khan, A. A., Kuang, Y., Song, L \& Li, W. (2019). Dysregulation of neuron differentiation in an autistic savant with exceptional memory. Molecular Brain. 12 (91), 2-12. https://doi.org/10.1186/s13041-019-0507-7

Subramanyam, A. A., Mukherjee, A., Dave, M. \& Chavda, K. (2019). Clinical Practice Guidelines for Autism Spectrum Disorders. Indian J Psychiatry, 61 (2), 254-269. https://doi.org/10.3389/ fnins.2018.00304

Treffert, D. A. (2009). The savant syndrome: An extraordinary condition. A synopsis: Past, present, future. Philosophical Transactions of the Royal Society B: Biological Sciences, 364 (1522), 1351-1357. https://doi.org/10.1098/rstb.2008.0326

Treffert, D. A. (2014). Savant syndrome: Realities, myths and misconceptions. Journal of Autism and Developmental Disorders, 44 (3), $564-571$. https://doi.org/10.1007/s10803-013-1906-8

Yunta, J. A. M., Alonso, T. O., Lucas, A. F., Maestú, F. \& Baduell, M. P. (2003). El síndrome de savant: o idiot savant. Rev Neurol. 36 (1), $157-161$. 